\title{
TANGO: effect of tango Argentino on cancer-associated fatigue in breast cancer patients - study protocol for a randomized controlled trial
}

Shiao Li Oei ${ }^{1}$, Thomas Rieser ${ }^{2}$, Sarah Becker ${ }^{3}$, Jessica Groß ${ }^{3}$, Harald Matthes ${ }^{1,2,4}$, Friedemann Schad ${ }^{1,5}$ and Anja Thronicke ${ }^{1,2,6^{*}}$ [D

\begin{abstract}
Background: The majority of breast cancer patients suffer from persistent impairments after completion of their primary oncological therapy. Cancer-related fatigue (CRF) in particular is a multidimensional syndrome having a profound negative impact on the quality of life. To counter CRF symptoms, physical activities are suggested as first-line interventions, mind-body therapies have been shown to be effective, and music therapy can also reduce anxiety and stress in breast cancer patients. Tango therapy that combines various elements can have an impact on physical, psychological, and cognitive abilities and could therefore have a beneficial effect on breast cancer patients. The purpose of this study is to investigate whether a 6-week tango module is suited as a therapeutic approach for people after primary breast cancer therapy to favorably influence their quality of life, especially CRF levels.

Methods: Sixty patients with a diagnosis for stage I-III breast cancer 12-48 months before enrollment and with CRF (age > 18) will be recruited and randomized 1:1 to a tango or a waiting-list group. Movement concepts using elements of Argentine tango (self-awareness, musical and spatial perception, self-perception, playfulness, shared experience) will be examined with the participants during six consecutive weekly 1-h tango sessions. The primary outcome will be the improvement of CRF (German version of the Cancer Fatigue Scale), and the secondary outcomes will be the improvement in sleep quality (Pittsburgh Sleep Quality Index) and quality of life (EORTC-QLQ-C30). Patient-reported outcomes will be measured at baseline and 6 weeks later; follow-up will be performed 6, 12, and 24 months after baseline. An evaluation will be performed by means of descriptive data analyses.

Discussion: Argentine tango, as a music-based movement therapy, can influence different skills and may improve several outcomes. The therapeutic use of Argentine tango in the care of breast cancer patients has not yet been reported. It is anticipated that participants receiving the tango module will have improved CRF, sleep, and quality of life scores compared to a waitlist control.
\end{abstract}

\footnotetext{
* Correspondence: anja.thronicke@havelhoehe.de

${ }^{1}$ Research Institute Havelhöhe at the Hospital Gemeinschaftskrankenhaus Havelhöhe, Berlin, Germany

${ }^{2}$ Institute for Social Medicine, Epidemiology and Health Economics, Charité Universitätsmedizin Berlin, Freie Universität Berlin, Humboldt-Universität zu Berlin, Berlin Institute of Health, Berlin, Germany

Full list of author information is available at the end of the article
}

(c) The Author(s). 2021 Open Access This article is licensed under a Creative Commons Attribution 4.0 International License, which permits use, sharing, adaptation, distribution and reproduction in any medium or format, as long as you give appropriate credit to the original author(s) and the source, provide a link to the Creative Commons licence, and indicate if changes were made. The images or other third party material in this article are included in the article's Creative Commons licence, unless indicated otherwise in a credit line to the material. If material is not included in the article's Creative Commons licence and your intended use is not permitted by statutory regulation or exceeds the permitted use, you will need to obtain permission directly from the copyright holder. To view a copy of this licence, visit http://creativecommons.org/licenses/by/4.0/ The Creative Commons Public Domain Dedication waiver (http://creativecommons.org/publicdomain/zero/1.0/) applies to the data made available in this article, unless otherwise stated in a credit line to the data. 
Trial registration: German Clinical Trials Registry (DRKS) DRKS00021601. Retrospectively registered on 21 August 2020

Keywords: Breast cancer, Dance, Fatigue, Insomnia, Health-related quality of life

\section{Administrative information}

Note: The numbers in curly brackets in this protocol refer to SPIRIT checklist item numbers. The order of the items has been modified to group similar items (see http://www.equator-network.org/reporting-guidelines/ spirit-2727-statement-defining-standard-protocol-itemsfor-clinical-trials/).

\begin{tabular}{|c|c|}
\hline Title $\{1\}$ & $\begin{array}{l}\text { TANGO - Effect of Tango Argentino } \\
\text { on cancer-associated fatigue in } \\
\text { breast cancer patients: Study Proto- } \\
\text { col for a Randomized Controlled } \\
\text { Trial }\end{array}$ \\
\hline Trial registration $\{2 \mathrm{a}$ and $2 \mathrm{~b}\}$. & $\begin{array}{l}\text { Trial registration number } \\
\text { DRKS00021601. Retrospectively } \\
\text { registered on } 21 \text { August } 2020 \text {. }\end{array}$ \\
\hline Protocol version $\{3\}$ & $\begin{array}{l}\text { The current protocol version is version } \\
\text { 2.0, dated from } 23 \text { March } 2020 \text {. }\end{array}$ \\
\hline Funding $\{4\}$ & $\begin{array}{l}\text { The primary sponsor is the } \\
\text { Forschungsinstitut Havelhöhe (FIH) } \\
\text { gGmbH am Gemeinschaftskrankenhaus } \\
\text { Havelhöhe. }\end{array}$ \\
\hline Author details $\{5 a\}$ & $\begin{array}{l}\text { SLO, MH, FS, AT: Research Institute } \\
\text { Havelhöhe gGmbH at Hospital } \\
\text { Gemeinschaftskrankenhaus Havelhöhe, } \\
\text { Berlin, Germany; TR, HM, AT: Charité - } \\
\text { Universitätsmedizin Berlin, corporate } \\
\text { member of Freie Universität Berlin and } \\
\text { Humboldt-Universität zu Berlin, Institute } \\
\text { of Social Medicine, Epidemiology and } \\
\text { Health Economics, Berlin, Germany; SB, } \\
\text { JG: Hospital Gemeinschaftskrankenhaus } \\
\text { Havelhöhe Breast Cancer Centre, Berlin, } \\
\text { Germany; MH: Medical Clinic for Gastro- } \\
\text { enterology, Infectiology and Rheuma- } \\
\text { tology CBF, Charité - } \\
\text { Universitätsmedizin Berlin, corporate } \\
\text { member of Freie Universität Berlin and } \\
\text { Humboldt-Universität zu Berlin, MH, FS: } \\
\text { Hospital Gemeinschaftskrankenhaus } \\
\text { Havelhöhe, Interdisciplinary Oncology } \\
\text { and Palliative Care, Berlin }\end{array}$ \\
\hline $\begin{array}{l}\text { Name and contact } \\
\text { information for the trial } \\
\text { sponsor }\{5 b\}\end{array}$ & $\begin{array}{l}\text { Research Institute Havelhöhe gGmbH at } \\
\text { Hospital Gemeinschaftskrankenhaus } \\
\text { Havelhöhe, Berlin, Germany } \\
\text { Trial's principal investigator: Friedemann } \\
\text { Schad, fschad@havelhoehe.de; scientific } \\
\text { coordinator: Anja Thronicke, anja. } \\
\text { thronicke@havelhoehe.de }\end{array}$ \\
\hline Role of sponsor $\{5 \mathrm{c}\}$ & $\begin{array}{l}\text { The sponsor is non-commercial. The } \\
\text { sponsor ensures quality management, } \\
\text { qualified and trained personnel, study } \\
\text { protocol compliance, submission of } \\
\text { relevant study documents to the ethics } \\
\text { committee and regulatory authorities. } \\
\text { He supports the study with trial unit fa- } \\
\text { cilities and study nurses. }\end{array}$ \\
\hline
\end{tabular}

\section{Introduction}

\section{Background and rationale $\{6 \mathrm{a}\}$}

Breast cancer still is the most common cancer in women worldwide [1]. About one-third of breast cancer patients experience moderate to severe fatigue symptoms $[2,3]$. Cancer-related fatigue (CRF) is a multidimensional syndrome having a profound negative impact on healthrelated quality of life (HRQL) and is a very prevalent and distressing side effect among breast cancer patients, which may often persist for many years after oncological treatment $[2,4]$. In a systematic review, it was found that an ameliorative benefit on HRQL can be reached with physical activity interventions such as yoga, physical selfmanagement, complementary exercises, art therapies, mind-body exercise therapies, and cognitive-behavioral therapies [5]. Currently, there is convincing evidence that specific doses of aerobic and/or resistance training could improve common cancer-related health outcomes, including fatigue, and it is recommended that physical activities are proposed as a first-line intervention for improving HRQL and counteracting CRF symptoms $[6,7]$. Mindfulness-based interventions, such as meditation and yoga, in particular, have been shown to be effective in reducing CRF levels [8]. Unfortunately, a lack of motivation and fatigue and a lack of time are often barriers to physical activity [9]. Several results from systematic reviews reveal mindfulness-based art therapy improves anxiety, depression, fatigue, stress, and HRQL [10-12]. Especially, cancer-related cognitive complaints such as fatigue, insomnia, and psychological distress might be managed through multimodal approaches. Another form of exercise therapy, which includes cognitive, emotional, and volitional elements, is eurythmy therapy [13], and interestingly, a multicenter study of 126 breast cancer patients reported that a multimodal approach that included eurythmy therapy and psychoeducation resulted in an improvement in HRQL and a significant reduction in symptoms of fatigue $[14,15]$. Also, behavioral techniques appear to play an important role in influencing psychosocial functioning and cognitive impairments [16]. Data of a systematic review found a positive role of life review on spiritual well-being, overall distress, and HRQL in patients with terminal or advanced cancer [17], which is important not only at the end of life. For instance, we reported that in breast cancer patients, elaborate consultations and life reviews conducted at first diagnosis were associated with a significant reduction in fatigue symptoms and relevant improvements in HRQL [18, 19]. Music therapy is another suitable 
creative arts treatment for improving psychological and physical outcomes in cancer patients, and a beneficial effect on anxiety and especially a moderate effect on fatigue and HRQL were found [20]. Hence, dance as a combination of physical activity, music, and mindful elements might be an appropriate and effective approach, to address CRF and to improve HRQL [21]. Protocols for randomized controlled trials of dance therapies for breast cancer patients have been published, to investigate different and various aspects. For example, it has been investigated whether dance therapy can relieve symptoms and stress in breast cancer patients, during radiation therapy [22], and whether traditional Greek dance [23] or belly dance [24] shows physical or psychological benefits. Furthermore, a pilot trial of 31 female cancer survivors and their partners examined ballroom dance, to improve their HRQL [25]. With a dance program for patients with breast cancer across five European countries, positive changes on HRQL and improvements of emotional and social scales were observed [26], and in a feasibility study, the efficacy of a balance training program using elements of Argentine tango for cancer survivors was assessed [27]. Argentine tango is a music-based movement therapy which can influence physical, psychological, and cognitive skills. In addition, it meets women's desire for social interaction [28]. Tango as a dance form has the advantage that it can be danced improvisationally in a variety of ways: without former experience, in changing roles, paired or in groups, as well as with or without physical contact. Interestingly, a systematic review revealed that Argentine tango has a tendency for positive effects on fatigue and HRQL in Parkinson's disease patients [29]. To date, no results of high-quality randomized controlled trials have been published on the effectiveness of dance therapies for cancer survivors. The aim of this study is to investigate whether therapeutic tango can improve selfreported quality of life, particularly fatigue levels, in breast cancer survivors.

\section{Objectives $\{7\}$}

This study protocol describes a randomized controlled trial for patients with breast cancer and increased CRF receiving either Argentine tango in a tango group or no Argentine tango in a waiting-list control group. The primary outcome measure is the change of CRF levels after 6 weeks. (A) The CRF changes of patients of the tango group will be compared to the waiting-list control group, and (B) prior to and post the 6-week Argentine tango treatment, the CRF changes are evaluated for the whole study cohort. The primary hypothesis is that a module consisting of six consecutive weekly 1-h Argentine tango lessons will promote a significant reduction of fatigue symptoms. Secondary objectives are the improvement of self-reported sleep quality and HRQL, prior to and post the Argentine tango treatment, and to explore the effects after 6,12 , and 24 months follow-up.

\section{Trial design $\{8\}$}

This is a single-center, prospective, two-arm randomized controlled trial designed to assess the effects of Argentine tango for people with breast cancer and CRF. The participants will be randomized to either the tango group or the waiting-list control group (Fig. 1). Evaluation of the patient-reported outcomes CRF, sleep quality, and HRQL will be performed.

\section{Methods: participants, interventions, and outcomes \\ Study setting $\{9\}$}

This study is conducted by the Forschungsinstitut Havelhöhe (FIH) at the hospital Gemeinschaftskrankenhaus Havelhöhe in Berlin (GKHB). Participants are recruited at the primary care facility of the Breast Cancer Centre at the GKHB.

\section{Eligibility criteria $\{10\}$ Inclusion, exclusion, and dropout criteria for participants}

Participants will be eligible to join the study if they had been diagnosed with breast cancer, have increased levels of fatigue, are willing to participate in this study, and if they meet all following criteria:

- Signed written informed consent

- Age $\geq 18$ years

- Stage I-III breast cancer has been diagnosed 12-48 months before enrollment

- Increased levels of fatigue ( $\geq 12$ points in the CFS-D questionnaire)

- Eastern Cooperative Oncology Group (ECOG) performance status $0-2$

Patients will not be eligible for the study if they meet one or more of the following exclusion criteria:

- No fatigue at all ( $\leq 12$ points in the CFS-D questionnaire)

- Pregnancy or breastfeeding

- Occurrence of recurrences or metastases

- Concurrent participation in other clinical trials

- Linguistic, medical, psychiatric, cognitive, or other conditions that may compromise the patient's ability to understand the patient information, comply with the study protocol, or complete the study

Participants must attend at least four of the six tango sessions and answer the questionnaires; if they do not, they will be considered dropouts. 


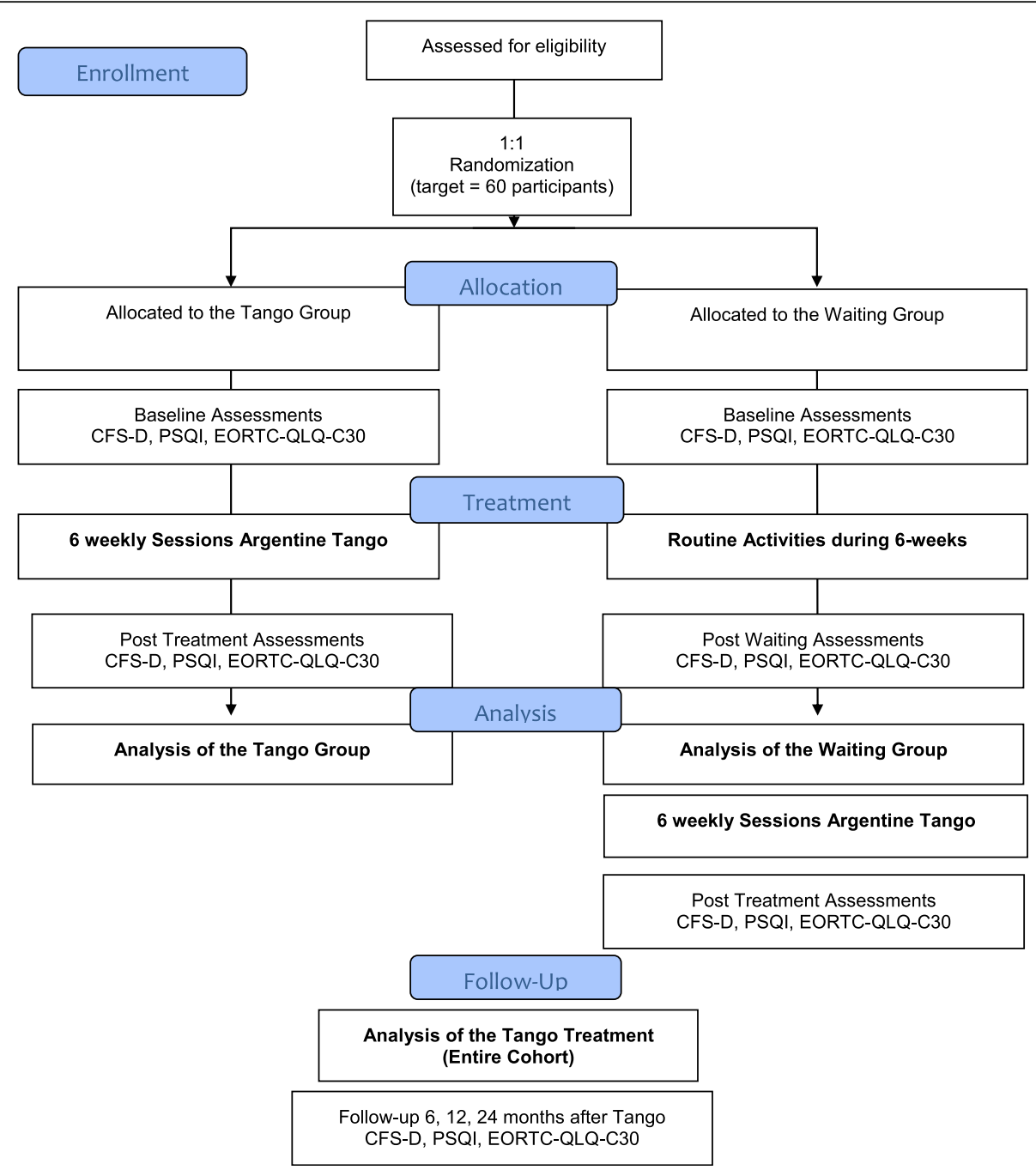

Fig. 1 Flow diagram of the study participants according to the Consolidated Standards of Reporting Trials (CONSORT 2010), illustrating the participant's timeline

Who will take informed consent? $\{26 \mathrm{a}\}$

It is the responsibility of the study physician to provide sufficient verbal and written information on the study's purpose and procedures, information on data protection procedures, possible advantages and disadvantages of participation, and option to withdraw from the study at any time and without any given reason and to take informed consent. Written informed consent will be obtained from all participants prior to study enrollment.

\section{Additional consent provisions for collection and use of participant data and biological specimens $\{26 \mathrm{~b}\}$}

The consent form asks whether participants agree that the data collected may be shared and published in a pseudonymized form for research purposes.

\section{Interventions}

Explanation for the choice of comparators $\{6 b\}$

The comparator is a waiting-list control group. All participants will be randomized to either the tango group or the waiting group. The participants of the waiting group will continue routine activities during the 6-week waiting period. During follow-up, patients from the waiting-list control group will receive the same Argentine tango module as the participants of the tango group. The follow-up assessments for both trial arms will be the same (Fig. 1).

\section{Intervention description $\{11 a\}$}

The Argentine tango program consists of six weekly 1-h tango sessions, which are conducted by a professional tango teacher and will be carried out in small groups of 
three to eight participants. Elements of movement and music of Argentine tango will be presented. The coordination, rhythm, and body awareness will be stimulated with harmonic movements to the tango music. Introductory exercises are practiced first without musical accompaniment and then with Argentine tango music. The 6-week program comprises breathing and relaxation exercises, balance finding, and includes tango walking and dancing elements as well as social interaction. The therapist will give explanations and lead guided exercises which are oriented towards walking to music, self-perceptions, and spatial perceptions as outlined in Table 1. Each group session will be structured in a warm-up, main part, and focused ending. The warm-up consists of a standing exercise with the feet open, with the imagination of moving through the body from the toes to the scalp and bringing awareness to the bodily sensations. The main parts have different focuses throughout the six sessions and include theoretical and practical instructions of walking and dancing techniques, repetitions with individual breaks, and explanations as needed (for details see Table 1). Each session ends with a 3-min standing exercise without movement, in silence with feet open and eyes closed.

\section{Criteria for discontinuing or modifying allocated interventions $\{11 b\}$}

Participants are encouraged to report any discomfort and to perform only movements that do not cause them any problems. If a participant does not attend a session, she is contacted by phone and asked how she is feeling, and options are sought to continue her tango sessions.
Adapting to the current COVID-19 pandemic conditions, the tango sessions are conducted in compliance with the national security and hygienic regulations, i.e., all dance movements, including paired actions, will be performed at a distance of $2 \mathrm{~m}$ to each other, the tango sessions will be conducted in a large therapy room with adequate oxygen ventilation, and the patients as well as the teacher are wearing surgical masks during the session. Before entering the therapy room, the teacher and the patients have to disinfect their hands with adequate disinfection detergent (including $>75 \%$ ethanol). Protective policies are constantly adapted to the locally applicable regulations; currently, participants must be vaccinated or have a valid negative SARS-CoV2 test to participate in Tango sessions.

\section{Strategies to improve adherence to interventions $\{11 \mathrm{c}\}$}

At the beginning of each session, participants are asked about their feelings with the previous session and experiences, and their preferences are discussed in the group. In case of any problems with adhering to the tango lesson, ways will be sought to carry out at least minimal essential parts of the treatment. The number of sessions each participant actually attended will be recorded. For the follow-up surveys, questionnaires will be sent with prepaid return envelopes, and if necessary, participants will be reminded by phone call to fill in and return them.

\section{Relevant concomitant care permitted or prohibited during the trial $\{11 d\}$}

Concomitant medications including nutritional supplements, vitamins, and natural remedies will be registered in the case

Table 1 Six-week Argentine tango protocol for people with breast cancer and fatigue

Session Description of the activities

$1 \quad$ Self-awareness. Warming-up (standing exercise, open feet, imaging of moving through the body from toes to scalp bringing awareness to patient's bodily sensations); axis and muscle tone; tension and relaxation; introduction of the tango position with feet closed, reaching in six directions (floor, sky, front, back, left, right); walking techniques, steps in four directions (forward, backward, left, right); walking forward in the line of dance with music; walking backward in the line of dance with music; changing directions in the line of dance from forward to backward while walking with music; and focused ending (standing exercise in silence with open feet and eyes closed, no movement)

2 Spatial and musical perception. Warming-up; walking technique, steps in four directions; walking in the line of dance to the music; changing directions in the line of dance from forward to backward while walking with music; musicality: double-beat, single-beat, and half-beat; focused ending

3 Social Perception. Warming-up; walking technique, steps in four directions; walking in the line of dance to the music; changing directions in the line of dance from forward to backward while walking with music; musicality: double-beat, single-beat, and half-beat; partner exercises "mirror dance" = no physical contact, distance $2 \mathrm{~m}$; mirror dance in a circle: one person leads the whole group; mirror dance in couples with changing partners walking in squares and walking in the line of dance with music; and focused ending

$4 \quad$ Playfulness. Warming-up; introduction of the "pivot" = turning on one foot; combining walking and pivot; mirror dance (distance $2 \mathrm{~m}$, no physical contact) in couples with changing partners with playful variations of walking together in the line of dance with music and with pivots; and focused ending

5 Sensation. Warming-up; mirror dance in couples (distance $2 \mathrm{~m}$, no physical contact) with changing partners with the elements of the previous four sessions; focus on the quality of movement and connection = not how we move matters, but how we feel moving; and focused ending

6 Shared experience. Warming-up; dancing summary of the five sessions; focus on communal dance experience (distance $2 \mathrm{~m}$, no physical contact); combining oneself with the group; and focused ending 
report form. Standard routine care and drugs as endocrine treatments do not affect patients' eligibility to enter or patients' participation during the trial.

\section{Provisions for post-trial care \{30\}}

After the whole tango module, participants will be provided with information about appropriate online tango exercises and classes that they could attend on a self-pay basis.

\section{Outcomes $\{12\}$}

Outcome measures are 1. the change of CRF levels, 2. the change of sleep quality, and 3. the change of HRQL.

1. CRF levels will be investigated with the German version of the certified and validated Cancer Fatigue Scale (CFS-D) [30]. It consists of a 15 -item questionnaire on three subscales (physical, cognitive, and affective fatigue), based on a 5-point Likert scale with a possible range of 0 (no fatigue) to 60 (maximum fatigue) [30]. The CFS-D is highly reliable with a robust validity and classifies values $\geq$ 30 points as clear symptoms of fatigue, $\geq 24$ points as suspected moderate fatigue, and $\leq 23$ points as only minor or no fatigue symptoms. A decline of scores indicates an improvement of fatigue while higher scores represent a higher degree of fatigue symptoms.

2. Self-reported sleep quality will be evaluated by the certified and validated Pittsburgh Sleep Quality Index (PSQI) questionnaire [31]. This questionnaire is composed of seven sleep-related subscales (subjective quality, latency, duration, habitual efficiency, disturbances, use of sleeping medication, and daytime sleepiness) all scored between 0 and 3 . The global PSQI sum scores range from 0 (no sleep disturbance) to 21 (maximally disturbed sleep), while a PSQI score greater than 5 classifies poor sleepers.

3. Self-reported HRQL will be investigated with the European Organization for Research and Treatment of Cancer Questionnaire C30 (EORTC-QLQ-C30) [32], which is structured into 15 different subscales (1 global health, 5 functional, and 9 symptoms, including fatigue and insomnia scales). Equations will be made as described in the EORTC-QLQ-C30 manual. The EORTC scores range from 0 to 100, and higher scores represent a better self-reported level in the functional dimensions but a higher degree of symptom burden.

Baseline information on diagnosis, such as histology, tumor classification, and potential previous treatments, will be collected from the clinical interview at study inclusion and the participants' medical records. All participants will be asked to answer all questionnaires at baseline, prior to and post-treatment, or waiting, and likewise in the framework of mid- and long-term surveys, 6,12 , and 24 months after completing the tango module (Fig. 1). Follow-up surveys will also ask about interim experiences with dance and movements, what medications were taken, and whether there are interests in continuing and online interventions. The courses of fatigue, sleep quality, and HRQL of participants of this study will be compared to those of previous patients of the GKHB Breast Cancer Center [18, 33].

\section{Participant timeline $\{13\}$}

In Fig. 1, trial procedures from enrollment to the end of the trial are illustrated.

\section{Sample size $\{14\}$}

The expected longitudinal differences between the means of the outcomes assessed will be estimated based on our previously published breast cancer studies [18, 33]. Previously, significant associations with medium effect sizes between non-pharmacological interventions and an improvement in fatigue were observed, and we found a mean difference of $7-12 \%$ [33]. For the characterization of the group and longitudinal differences, Student's $t$-tests and Pearson's chi-squared tests with Yates' continuity correction were performed. For the calculation of the sample size, medium to large effect size differences (Cohen's $d$ of 0.8 ) and a power of $80 \%$, as well as a significant level of $5 \%$ (two-sided) for the primary outcome fatigue, are assumed. Thus, a case number of 25 patients per group will be necessary. Assuming a dropout rate of $20 \%$, we need to recruit and randomize 60 participants.

\section{Recruitment $\{15\}$}

Breast cancer patients who have been treated at the GKH Breast Cancer Center and who have given their consent to be contacted for clinical studies will be briefly screened for eligibility, contacted by phone call, and invited to participate in this study. A clinical interview is scheduled with interested and eligible patients. After obtaining written informed consent and after the patient's enrollment in this study, sociodemographic and clinical data (age, body mass index, tumor stage, hormonal status, concomitant medications, sports activities) are surveyed, and questionnaires will be given to participants.

\section{Assignment of interventions: allocation Sequence generation $\{16 a\}$}

The randomization sequence has been created [34] using the website www.jerrydallal.com/random/random 
block_size_r.htm with a 1:1 allocation, using block sizes of four to six. The randomization plan for subjects no. 1-40 was generated on 12 May 2020 (Seed No. 15277) and for subjects no. 41-60 on 2 March 2021 (Seed No. 15434).

\section{Concealment mechanism \{16b\}}

In the temporal order of the study inclusion, allocation will be made according to the randomization plan to either the tango or the waiting group. Participants, physicians, and therapists are blinded to the randomization and allocation processes and will have no influence on the assignment of participants to each of both groups.

\section{Implementation $\{16 c\}$}

In a clinical interview, a study physician collects patient clinical and demographic data and provides information about the study. All patients who give their written consent to participate in the study will be enrolled in this study. Unique identifiers were generated using an ID-generator software [35]. A study investigator will register the participants, using the randomization plan to allocate to either the tango or waiting group. According to the availability to participate in a tango course, the participants are asked to complete and return the questionnaires at specified dates. The flow diagram of the study design is depicted in Fig. 1.

All patient data is handled in accordance with the General Data Protection Regulation (GDPR). All data from the participants will be maintained confidentially before, during, and after the trial and is stored securely at the study site, with limited access.

\section{Assignment of interventions: blinding \\ Who will be blinded $\{17 a\}$}

All participants, physicians, and therapists are blinded to the randomization and allocation process. Due to the nature of the study design, participants are not blinded to the tango treatment itself; however, as the waiting-list control group will as well receive the same tango lessons later on, they will not know whether they are in the waiting list or not. According to their allocation, study participants will be individually requested by the members of the research team to complete the questionnaires. The flow diagram of the study design is depicted in Fig. 1.

Procedure for unblinding if needed $\{17 b\}$

There is no need of unblinding.

\section{Data collection and management}

Plans for assessment and collection of outcomes $\{18 a\}$

The primary outcome is change in CRF measured with the validated CFS-D questionnaire [30]. The secondary outcomes are sleep quality, measured with the Pittsburgh Sleep Quality Index (PSQI) [31], and patient's HRQL, measured with the European Organization for Research and Treatment of Cancer Questionnaire C30 (EORTC-QLQ-C30) [32].

After obtaining written informed consent and inclusion in this study, sociodemographic and clinical data are surveyed, and questionnaires will be given to participants. All questionnaires will be collected prior to and post-treatment and during follow-up 6, 12, and 24 months after the start of the tango module (Fig. 1).

\section{Plans to promote participant retention and complete follow-up $\{18 b\}$}

If there are problems with participation in a tango session, a phone call will be made to find ways to continue attendance. For the follow-up surveys, participants will be contacted by postal mail, by stamped return envelopes enclosed, and by personal telephone calls.

\section{Data management $\{19\}$}

Pseudonymized data will be collected with paper case report forms and paper-based questionnaires. All essential trial documentation will be kept within the Trial Master File. For all collected data, pseudonymized data entry is performed at the study center by members of the research staff [36, 37]. Clinical and demographic data will be retrieved from the patients during the informed consent interview by the study physician.

\section{Confidentiality $\{27\}$}

Data management and processing of the pseudonymized collected data are subject to the General Data Protection Regulation (GDPR). In order to protect the confidentiality, the pseudonymized collected data will be stored for 15 years after the end of this study in a pseudonymized form at the study center of the FIH at the GKHB. The pseudonymized data may only be reviewed by authorized persons of the research team. No third parties will have access to these data.

Plans for collection, laboratory evaluation, and storage of biological specimens for genetic or molecular analysis in this trial/future use $\{33\}$

No biological specimens will be collected. 


\section{Statistical methods}

Statistical methods for primary and secondary outcomes $\{20 \mathrm{a}\}$

All statistical analyses will be performed using Excel 2010 (Microsoft, USA) and the software $R$ ( $R$ version 4.0.5) [38]. Baseline data will be summarized using descriptive statistics. Continuous variables will be described as means with standard deviation and median with interquartile range (IQR), categorical variables will be summarized as frequencies and percentages. Student's $t$-tests will be applied, and $p$-values $<0.05$ are considered to be significant. For the characterization of group differences, Pearson's chi-squared tests with Yates' continuity correction and Student's $t$-tests will be performed. The detection of longitudinal effects will be characterized with Student's $t$-tests and to identify influencing factors and to address potential sources of bias and potential confounders, and adjusted multivariable linear regression analyses will be performed. In order to yield reliable model results, stepwise regression selections will be performed, and models with high adjusted $R^{2}$ will be chosen. According to Cohen's interpretation [39], $R^{2}$ values between 13 and $25 \%$ indicate medium, and $R^{2}$ values $26 \%$ or above indicate high effect sizes. In addition to statistical significance, we will report descriptive statistics and effect size estimates.

\section{Interim analyses $\{21 \mathrm{~b}\}$}

No interim analysis will be performed. No unblinded interim results have been and will not be presented anywhere.

\section{Methods for additional analyses (e.g., subgroup analyses) \{20b\}}

Subgroup analyses will be performed regarding the possible confounders such as concurrent endocrine treatment, concomitant medications for symptom relief, and sport activity. Multilevel modeling can be used for subgroup analyses and to account for confounding variables. Confounding variables (age, body mass index, tumor stage, hormonal status, endocrine treatment, concomitant medication, sport activity, and the respective outcome values at baseline) will be considered. Furthermore, adjusted multivariable linear regression analyses will be performed, to address potential confounders and influencing factors.

\section{Methods in analysis to handle protocol non-adherence} and any statistical methods to handle missing data $\{20 \mathrm{c}\}$ Patients who are unable to participate in the tango treatment or withdraw consent to participate in the study will be excluded and not replaced. If individual sessions cannot be attended, this will be noted, and the surveys will continue as planned. Regarding the evaluation of the questionnaires, we follow the EORTCQLQ-C30 scoring manual on the calculation and handling of missing data. We do not plan to use imputation when reporting outcome data.

Plans to give access to the full protocol, participant leveldata, and statistical code $\{31 \mathrm{c}\}$

The trial protocol and anonymized data on the group level may be shared with scientists who have medically or scientifically well-founded reasons; data protection according to GDPR and ethics according to ethical approval must be ensured.

\section{Oversight and monitoring Composition of the coordinating center and trial steering committee $\{5 d\}$}

The trial coordination team meets regularly with the chief investigator, study physicians, tango teacher, and further research team members. The study design, study protocol, data management, and all study-related documents are critically reviewed. The coordinating investigator is responsible for the trial registration, revisions of the study protocol and application/amendments to the ethics committee, and scheduling of regular team meetings. All involved investigators and team members ensure compliance with the study protocol and ensure the follow-up according to the protocol. The steering committee consisting of clinical experts and sponsor's staff is not directly involved in the study and ensure the scientific quality of the study, the study protocol, and the study report.

Composition of the data monitoring committee, its role, and reporting structure $\{21 a\}$

Not applicable, this trial is not monitored.

\section{Adverse event reporting and harms $\{22\}$}

During each tango session, information on the occurrence of adverse events is regularly retrieved and documented. Adverse events related to the tango treatment during the 6-week sessions will be recorded by the coordinating investigator.

\section{Frequency and plans for auditing trial conduct $\{23\}$}

The trial office monitors the aspects of the study on an ongoing basis, and no audits are planned. The Trial Steering Committee will meet every 5-7 months.

\section{Plans for communicating important protocol amendments to relevant parties (e.g., trial participants, ethical committees) $\{25\}$}

All protocol deviations or modifications will be documented by appropriate updates in due course and according to the national regulations; all substantial 
protocol amendments will be communicated to the ethics committee of the Medical Association Berlin (Berlin Ethik-Kommission der Ärztekammer Berlin) and German clinical trial register, DRKS.

\section{Dissemination plans $\{31$ \}}

The study results will be presented in conferences and symposia and submitted for publication in relevant medical journals. Portions from this study will also be included in two doctoral theses.

\section{Discussion}

Here, we present a study protocol on the efficacy of a 6 week Argentine tango module for the reduction of CRF symptoms in people after primary breast cancer treatment. A systematic review and meta-analyses revealed that supervised aerobic exercise in particular was statistically more effective than conventional care in improving CRF in breast cancer patients [40]. As has been reported, multimodal supportive strategies such as mind-body therapies including relaxation techniques, stress management, meditation, physical activity [41], and also physical self-management interventions [42] have been established to counteract adverse effects of oncological therapeutics and seem to generate beneficial effects on perceived HRQL including fatigue levels $[8,43]$. Some of these interventions have been included in international guideline recommendations $[8,44,45]$. Currently, it is widely accepted that exercise and physical activity are effective to reduce CRF levels [7], and most breast cancer patients are aware of this and also want to be active, but lack the necessary impulses to do so, especially when having fatigue symptoms. In the past, most studies have addressed HRQL of breast cancer patients during their oncology treatments, and more studies are needed that address symptom burden in breast cancer survivors who have successfully completed their oncology treatments [5]. Therefore, we decided to offer a treatment program that can fill this gap, is not too elaborate and strenuous, and can be performed even with physical limitations.

CRF is a multidimensional syndrome, frequently occurring with other neuropsychological symptoms also acting at sleep/wake centers in the brain [46]. Furthermore, it has been observed that CRF frequently occurs alongside and is interrelated with sleep disturbances [47]. Interventions that affect the biorhythm may therefore also influence the mood and well-being, as well as might modulate fatigue symptoms of cancer patients. Dance can be understood as a multimodal treatment based on body awareness, expression, and rhythm, combining both psychotherapeutic therapy and physical activity, and can support emotional, cognitive, and spiritual integration [48]. A recent study reported that ballroom dancing may improve functional exercise capacity and may be associated with a high selfefficacy and active lifestyle [49]. However, no effects of this dance training on fatigue symptoms were observed, but there was a limitation to individuals who had a healthy dance partner and presumably exhibited only mild fatigue symptoms, since data were collected only from participants of active dance classes [49]. To address people with breast cancer who suffer from CRF, have low self-confidence, are less fit, and often do not have any appropriate partner for dance exercises, we chose Argentine tango, a dance style that allows adaptation to different conditions and can be performed even under the current pandemic situation by dancing with a surgical mask and in a distance of $2 \mathrm{~m}$. The therapeutic use of Argentine tango to reduce fatigue symptoms in breast cancer patients has not yet been assessed. Argentine tango comprises relaxation techniques, supports balance finding and self- and spatial perception exercises, and thus may influence physical, psychological, and cognitive skills as well as probably also fatigue levels and insomnia. It is expected that receiving the 6-week tango module will significantly reduce fatigue and insomnia symptoms in our breast cancer patients. Previously, we could show that the treatment of breast cancer patients with chemotherapy is associated with a severe increase in fatigue, in particular, of cognitive fatigue [50]. There are hardly any therapeutic options to alleviate such cognitive impairments. It has been reported that Argentine tango can influence and improve cognitive abilities in Parkinson's patients [29]. Therefore, it is believed that the tango may also have a positive effect on cognitive fatigue in breast cancer patients. The tango group is expected to have a clinically relevant reduction in fatigue and insomnia symptoms compared to the waitlist control. Further benefits related to aspects of HRQL may be observed. In addition, follow-up surveys of the entire cohort will be conducted at 6,12 , and 24 months to examine how this tango treatment will be perceived by the participants and how lasting the effects prove to be.

\section{Trial status}

The current protocol version is 2.0, dated from 23 March 2020. The trial is ongoing and currently enrolling. The first participant was enrolled on 10 June 2020, and recruitment is expected to be finalized in the second quarter of 2022 with the follow-up completed by the end of March 2024.

\footnotetext{
Abbreviations

CRF: Cancer-related fatigue; CFS-D: German version of the Cancer Fatigue Scale; ECOG: Eastern Cooperative Oncology Group; EORTC: European Organisation for Research and Treatment of Cancer; FIH: Forschungsinstitut Havelhöhe; GDPR: General Data Protection Regulation; GKHB: Gemeinschaftskrankenhaus Havelhöhe Berlin; HRQL: Health-related quality of life; PSQI: Pittsburgh Sleep Quality Index
} 


\section{Acknowledgements}

We would like to acknowledge Thomas Rieser and Sarah Becker for the acquisition of data alongside their doctoral theses. We also thank Irena Cop and all other medical documentation officers at the FIH and GKHB involved in supporting the present work.

\section{Authors' contributions $\{31 \mathrm{~b}\}$}

SLO conceived the study; initiated the study design; developed the methodology; was responsible for the data curation, formal analysis, validation, and visualization; wrote the first draft of the manuscript; and reviewed it. TR conceived the study; initiated the study design; developed the methodology; was responsible for the validation, visualization, writing, and reviewing; and developed and implemented the tango protocol. SB conceived the study, initiated the study design, developed the methodology, and was responsible for the validation, visualization, writing, reviewing, and recruitment of participants. JG conceived the study, initiated the study design, developed the methodology, and was responsible for the validation, writing, reviewing, and recruitment of participants. $\mathrm{MH}$ conceived the study, initiated the study design, developed the methodology, and was responsible for the validation, writing, and reviewing. FS is the principal investigator of the study; he conceived the study, initiated the study design, developed the methodology, and was responsible for the funding acquisition, validation, visualization, writing, reviewing, and project administration. AT is the scientific coordinator of the study; she conceived the study, initiated the study design, developed the methodology, and was responsible for the data curation, formal analysis, validation, visualization, writing, and reviewing. All authors commented on the drafts of the manuscript and read and approved the final manuscript.

\section{Funding $\{4\}$}

This study is supported via the institutional budget of the $\mathrm{FIH}$, which is the primary sponsor of the study.

\section{Availability of data and materials $\{29\}$}

Any material required to support the protocol can be supplied on reasonable request.

\section{Declarations}

\section{Ethics approval and consent to participate $\{24\}$}

This study complies with the principles laid down in the Declaration of Helsinki [51] and was approved by the ethics of the Medical Association Berlin (Berlin_Ethik-Kommission der Ärztekammer Berlin) on 2 April 2020 with the reference number Eth-05/20. This trial was registered at the German Clinical Trials Register (Trial registration number DRKS00021601) on 21 August 2020. Written informed consent will be obtained from all participants prior to study enrollment. All substantial protocol deviations or modifications will be communicated to the Ethics Committee and German Clinical Trials Register.

\section{Consent for publication $\{32\}$}

Model consent forms will be provided on request.

\section{Competing interests $\{28\}$}

JG reports grants from Roche (honoraria for speaking). HM is a member of the board of directors of Weleda AG, a member of the Network Arbeitsgemeinschaft der Wissenschaftlichen Fachgesellschaften (AWMF e.V.) guideline committee for integrative oncology (Guideline for Complementary Medicine in the Treatment of Oncological Patients), and has an endowed professorship at the Charité Universitätsmedizin Berlin, which is financed by the Software AG Foundation, outside the submitted work. FS reports grants from Helixor Heilmittel GmbH (travel costs and honoraria for speaking), grants from AstraZeneca (travel costs and honoraria for speaking), grants from Abnoba $\mathrm{GmbH}$, and grants from Iscador AG, outside the submitted work. The other authors declared that they have no competing interests. No payment was received for any other aspects of the submitted work. There are no patents, products in the development, or marketed products to declare. There are no other relationships/conditions/circumstances that present a potential conflict of interest.

\section{Author details}

${ }^{1}$ Research Institute Havelhöhe at the Hospital Gemeinschaftskrankenhaus Havelhöhe, Berlin, Germany. ${ }^{2}$ Institute for Social Medicine, Epidemiology and Health Economics, Charité - Universitätsmedizin Berlin, Freie Universität Berlin, Humboldt-Universität zu Berlin, Berlin Institute of Health, Berlin, Germany. ${ }^{3}$ Breast Cancer Centre, Hospital Gemeinschaftskrankenhaus Havelhöhe, Berlin, Germany. ${ }^{4}$ Medical Clinic for Gastroenterology, Infectiology and Rheumatology CBF Charité - Universitätsmedizin Berlin, Freie Universität Berlin, Humboldt-Universität zu Berlin, Berlin Institute of Health, Berlin, Germany. ${ }^{5}$ Institute for Gastroenterology, Hospital Gemeinschaftskrankenhaus Havelhöhe, Berlin, Germany. ${ }^{6}$ Interdisciplinary Oncology and Palliative Care, Hospital Gemeinschaftskrankenhaus Havelhöhe, Berlin, Germany.

Received: 7 June 2021 Accepted: 23 November 2021

Published online: 02 December 2021

\section{References}

1. Bray F, Ferlay J, Soerjomataram I, Siegel RL, Torre LA, Jemal A. Global cancer statistics 2018: GLOBOCAN estimates of incidence and mortality worldwide for 36 cancers in 185 countries. CA: a cancer journal for clinicians. 2018; 68(6):394-424. PubMed PMID: 30207593. Epub 2018/09/13. eng. https://doi. org/10.3322/caac.21492.

2. Fabi A, Falcicchio C, Giannarelli D, Maggi G, Cognetti F, Pugliese P. The course of cancer related fatigue up to ten years in early breast cancer patients: what impact in clinical practice? Breast (Edinburgh, Scotland). 2017; 34:44-52. PubMed PMID: 28500901. Epub 2017/05/14. eng. https://doi.org/1 0.1016/j.breast.2017.04.012

3. Schmidt ME, Chang-Claude J, Seibold P, Vrieling A, Heinz J, Flesch-Janys D, et al. Determinants of long-term fatigue in breast cancer survivors: results of a prospective patient cohort study. Psychooncology. 2015;24(1):40-6. PubMed PMID: 24839264. Epub 2014/05/20. eng. https://doi.org/10.1002/ pon.3581.

4. Cella D, Peterman A, Passik S, Jacobsen P, Breitbart W. Progress toward guidelines for the management of fatigue. Oncology (Williston Park, NY). 1998;12(11A):369-77. PubMed PMID: 10028520. Epub 1999/02/24. eng.

5. Mokhatri-Hesari P, Montazeri A. Health-related quality of life in breast cancer patients: review of reviews from 2008 to 2018. Health Qual Life Outcomes. 2020;18(1):338. 33046106, PMC7552560. Epub 2020/10/14. eng. https://doi. org/10.1186/s12955-020-01591-x.

6. Mitchell SA, Hoffman AJ, Clark JC, DeGennaro RM, Poirier P, Robinson CB, et al. Putting evidence into practice: an update of evidence-based interventions for cancer-related fatigue during and following treatment. Clin J Oncol Nurs. 2014;18(Suppl):38-58. PubMed PMID: 25427608. Epub 2014/ 11/28. eng. https://doi.org/10.1188/14.CJON.S3.38-58.

7. Campbell KL, Winters-Stone KM, Wiskemann J, May AM, Schwartz AL, Courneya KS, et al. Exercise guidelines for cancer survivors: consensus statement from International Multidisciplinary Roundtable. Med Sci Sports Exerc. 2019;51(11):2375-90. PubMed PMID: 31626055. Epub 2019/10/19. eng https://doi.org/10.1249/MSS.0000000000002116.

8. Greenlee H, DuPont-Reyes MJ, Balneaves LG, Carlson LE, Cohen MR, Deng G, et al. Clinical practice guidelines on the evidence-based use of integrative therapies during and after breast cancer treatment. CA: a cancer journal for clinicians. 2017;67(3):194-232. 28436999, PMC5892208. Epub 2017/04/25. eng. https://doi.org/10.3322/caac.21397.

9. Frikkel J, Götte M, Beckmann M, Kasper S, Hense J, Teufel M, et al. Fatigue, barriers to physical activity and predictors for motivation to exercise in advanced Cancer patients. BMC Palliative Care. 2020 2020/03/31;19(1):43.

10. Cillessen L, Johannsen M, Speckens AEM, Zachariae R. Mindfulness-based interventions for psychological and physical health outcomes in cancer patients and survivors: a systematic review and meta-analysis of randomized controlled trials. Psychooncology. 2019;28(12):2257-69. 31464026, PMC6916350. Epub 2019/08/30. eng. https://doi.org/10.1002/pon.5214.

11. Sun $H$, Huang $H$, Ji S, Chen $X, X u Y$, Zhu F, et al. The efficacy of cognitive behavioral therapy to treat depression and anxiety and improve quality of life among early-stage breast cancer patients. Integr Cancer Ther. 2019;18: 1534735419829573. 30791739, PMC6432673. Epub 2019/02/23. eng. https:// doi.org/10.1177/1534735419829573.

12. Xunlin NG, Lau Y, Klainin-Yobas P. The effectiveness of mindfulness-based interventions among cancer patients and survivors: a systematic review and meta-analysis. Support Care Cancer. 2020;28(4):1563-78. PubMed PMID: 
31834518. Epub 2019/12/14. eng. https://doi.org/10.1007/s00520-01905219-9.

13. Kienle GS, Albonico HU, Baars E, Hamre HJ, Zimmermann P, Kiene H. Anthroposophic medicine: an integrative medical system originating in Europe. Global advances in health and medicine. 2013;2(6):20-31. 24416705, PMC3865373. Epub 2014/01/15. eng. https://doi.org/10.7453/gahmj.2012.087.

14. Kroz M, Reif M, Glinz A, Berger B, Nikolaou A, Zerm R, et al. Impact of a combined multimodal-aerobic and multimodal intervention compared to standard aerobic treatment in breast cancer survivors with chronic cancerrelated fatique - results of a three-armed pragmatic trial in a comprehensive cohort design. BMC cancer. 2017;17(1):166. 28253845, PMC5335840. Epub 2017/03/04. eng. https://doi.org/10.1186/s12885-017-3142-7.

15. Poier D, Bussing A, Rodrigues Recchia D, Beerenbrock Y, Reif M, Nikolaou A, et al. Influence of a multimodal and multimodal-aerobic therapy concept on health-related quality of life in breast cancer survivors. Integr Cancer Ther. 2019;18:1534735418820447. 30584782, PMC6432685. Epub 2018/12/26. eng. https://doi.org/10.1177/1534735418820447.

16. Duijts SF, Faber MM, Oldenburg HS, van Beurden M, Aaronson NK. Effectiveness of behavioral techniques and physical exercise on psychosocial functioning and health-related quality of life in breast cancer patients and survivors--a meta-analysis. Psychooncology. 2011;20(2):115-26. PubMed PMID: 20336645. Epub 2010/03/26. eng. https://doi.org/10.1002/ pon. 1728.

17. Wang CW, Chow AY, Chan CL. The effects of life review interventions on spiritual well-being, psychological distress, and quality of life in patients with terminal or advanced cancer: a systematic review and meta-analysis of randomized controlled trials. Palliat Med. 2017;31(10):883-94. PubMed PMID: 28488923. Epub 2017/05/11. eng. https:/doi.org/10.1177/0269216317705101

18. Oei SL, Thronicke A, Matthes H, Schad F. Assessment of integrative nonpharmacological interventions and quality of life in breast cancer patients using real-world data. Breast cancer (Tokyo, Japan). 2021 May;28(3):608-17. PubMed PMID: 33393018. Epub 2021/01/05. eng.

19. Thronicke A, Kroz M, Merkle A, Matthes H, Herbstreit C, Schad F. Psychosocial, cognitive, and physical impact of elaborate consultations and life review in female patients with non-metastasized breast cancer. Complement Med Res. 2018;25(2):92-101. PubMed PMID: 29656290. Epub 2018/04/16. eng. https://doi.org/10.1159/000486672.

20. Bradt J, Dileo C, Magill L, Teague A. Music interventions for improving psychological and physical outcomes in cancer patients. Cochrane Database Syst Rev. 2016;15(8):CD006911 27524661. Epub 2016/08/16. eng.

21. Sturm I, Baak J, Storek B, Traore A, Thuss-Patience P. Effect of dance on cancer-related fatigue and quality of life. Support Care Cancer. 2014;22(8): 2241-9 24671434. Epub 2014/03/29. eng.

22. Ho RT, Fong TC, Cheung IK, Yip PS, Luk MY. Effects of a short-term dance movement therapy program on symptoms and stress in patients with breast cancer undergoing radiotherapy: a randomized, controlled, singleblind trial. J Pain Symp Manage. 2016;51(5):824-31. PubMed PMID: 26975625. Epub 2016/03/16. eng. https://doi.org/10.1016/j.jpainsymman.201 5.12.332.

23. Kaltsatou A, Mameletzi D, Douka S. Physical and psychological benefits of a 24-week traditional dance program in breast cancer survivors. J Bodywork Movement Ther. 2011;15(2):162-7. PubMed PMID: 21419356. Epub 2011/03/ 23. eng. https://doi.org/10.1016/j.jbmt.2010.03.002.

24. Boing L, do Bem Fretta T, de Carvalho Souza Vieira M, Pereira GS, Moratelli J, Sperandio FF, et al. Pilates and dance to patients with breast cancer undergoing treatment: study protocol for a randomized clinical trial MoveMama study. Trials. 2020 Jan 7;21(1):35. PubMed PMID: 31910872. Pubmed Central PMCID: PMC6947954. Epub 2020/01/09. eng.

25. Pisu M, Demark-Wahnefried W, Kenzik KM, Oster RA, Lin CP, Manne S, et al. A dance intervention for cancer survivors and their partners (RHYTHM). J Cancer Survivorship. 2017;11(3):350-9. 28070770, PMC5419878 Epub 2017/01/11. eng.

26. Karkou V, Dudley-Swarbrick I, Starkey J, Parsons A, Aithal S, Omylinska-Thurston $J$, et al. Dancing with health: quality of life and physical improvements from an EU Collaborative Dance Programme with women following breast cancer treatment. Front Psychol. 2021;12:635578. 33716903, PMC7943865. Epub 2021/ 03/16. eng. https://doi.org/10.3389/fpsyg.2021.635578.

27. Worthen-Chaudhari L, Lamantia MT, Monfort SM, Mysiw W, Chaudhari AMW, Lustberg MB. Partnered, adapted argentine tango dance for cancer survivors: a feasibility study and pilot study of efficacy. Clinical biomechanics (Bristol, Avon). 2019 Dec;70:257-64. PubMed PMID: 31751861. Epub 2019/11/22. eng.
28. Witkos J, Hartman-Petrycka M. Implications of Argentine tango for health promotion, physical well-being as well as emotional, personal and social life on a group of women who dance. Int J Environ Res Public Health. 2021; 31(11):18(11). PubMed PMID: 34072683. Epub 2021/06/03. https://doi.org/1 0.3390/ijerph18115894

29. Lotzke D, Ostermann T, Bussing A. Argentine tango in Parkinson disease--a systematic review and meta-analysis. BMC Neurol. 2015;15(1): 226. 26542475, PMC4636067. Epub 2015/11/07. eng. https://doi.org/1 $0.1186 / s 12883-015-0484-0$

30. Kroz M, Zerm R, Reif M, HB VONL, Schad F, Bussing A, et al. Validation of the German version of the Cancer Fatigue Scale (CFS-D). Eur J Cancer Care. 2008;17(1):33-41. 18181889.

31. Buysse DJ, Reynolds CF 3rd, Monk TH, Berman SR, Kupfer DJ. The Pittsburgh Sleep Quality Index: a new instrument for psychiatric practice and research. Psychiatry Res. 1989;28(2):193-213. PubMed PMID: 2748771. Epub 1989/05/ 01. eng. https://doi.org/10.1016/0165-1781(89)90047-4.

32. Aaronson NK, Ahmedzai S, Bergman B, Bullinger M, Cull A, Duez NJ, et al. The European Organization for Research and Treatment of Cancer QLQ-C30: a quality-of-life instrument for use in international clinical trials in oncology. J Natl Cancer Institute. 1993;85(5):365-76. PubMed PMID: 8433390. Epub 1993/03/03. eng. https://doi.org/10.1093/jnci/85.5.365.

33. Schad F, Thronicke A, von Trott P, Oei SL. Analysis of changes in cancer-related fatigue of breast cancer patients receiving an integrative medicine program. Integr Cancer Ther. 2020;19:1534735420963780. 33040629, PMC7585890. Epub 2020/10/13. eng. https:/doi.org/10.1177/1534735420963780.

34. Kim J, Shin W. How to do random allocation (randomization). Clin Orthopedic Surg. 2014;6(1):103-9. 24605197, PMC3942596. Epub 2014/03/08. eng. https://doi.org/10.4055/cios.2014.6.1.103.

35. Olden M, Holle R, Heid IM, Stark K. IDGenerator: unique identifier generator for epidemiologic or clinical studies. BMC Med Res Methodol. 2016;16(1): 120. 27628043, PMC5024489. Epub 2016/09/16. eng. https://doi.org/10.1186/ s12874-016-0222-3.

36. Schad F, Axtner J, Happe A, Breitkreuz T, Paxino C, Gutsch J, et al. Network oncology (NO)--a clinical cancer register for health services research and the evaluation of integrative therapeutic interventions in anthroposophic medicine. Forschende Komplementarmedizin. 2013;20(5):353-60. 24200825. https://doi.org/10.1159/000356204.

37. Schad F, Thronicke A, Merkle A, Steele ML, Kroz M, Herbstreit C, et al. Implementation of an integrative oncological concept in the daily care of a German certified breast cancer center. Complement Med Res. 2018;25(2): 85-91. PubMed PMID: 29510405. Epub 2018/03/07. eng. https://doi.org/1 $0.1159 / 000478655$.

38. Team RC. R: a language and environment for statistical computing 2016

39. Cohen J. A power primer. Psychol Bulletin. 1992;112(1):155-9. PubMed PMID: 19565683. Epub 1992/07/01. eng. https://doi.org/10.1037/0033-2 909.112.1.155.

40. Meneses-Echavez JF, Gonzalez-Jimenez E, Ramirez-Velez R. Effects of supervised exercise on cancer-related fatigue in breast cancer survivors: a systematic review and meta-analysis. BMC Cancer. 2015;15(1):77. 25885168, PMC4364505. Epub 2015/04/18. eng. https://doi.org/10.1186/ s12885-015-1069-4.

41. Peterson LL, Ligibel JA. Physical activity and breast cancer: an opportunity to improve outcomes. Curr Oncol Rep. 2018;20(7):50. PubMed PMID: 29713835. Epub 2018/05/02. eng. https://doi.org/10.1007/s11912-0180702-1.

42. Van Dijck S, Nelissen P, Verbelen H, Tjalma W, Gebruers N. The effects of physical self-management on quality of life in breast cancer patients: a systematic review. Breast (Edinburgh, Scotland). 2016;28:20-8. PubMed PMID: 27173849. Epub 2016/05/14. eng.

43. Lipsett A, Barrett S, Haruna F, Mustian K, O'Donovan A. The impact of exercise during adjuvant radiotherapy for breast cancer on fatigue and quality of life: a systematic review and meta-analysis. Breast (Edinburgh, Scotland). 2017 Apr;32: 144-55. PubMed PMID: 28189100. Epub 2017/02/12. eng.

44. Fabi A, Bhargava R, Fatigoni S, Guglielmo M, Horneber M, Roila F, et al. Cancer-related fatigue: ESMO Clinical Practice Guidelines for diagnosis and treatment. Ann Oncol. 2020;31(6):713-23. PubMed PMID: 32173483. Epub 2020/03/17. eng. https://doi.org/10.1016/j.annonc.2020.02.016.

45. Lyman GH, Greenlee H, Bohlke K, Bao T, DeMichele AM, Deng GE, et al. Integrative therapies during and after breast cancer treatment: ASCO endorsement of the SIO Clinical Practice Guideline. J Clin Oncol. 2018; 36(25):2647-55 29889605. Epub 2018/06/12. eng. 
46. Bower JE, Lamkin DM. Inflammation and cancer-related fatigue: mechanisms, contributing factors, and treatment implications. Brain Behav Immun. 2013;30(Suppl):S48-57. 22776268, PMC3978020. Epub 2012/07/11. eng. https://doi.org/10.1016/j.bbi.2012.06.011.

47. Walker WH, 2nd, Borniger JC. Molecular mechanisms of cancer-induced sleep disruption. Int J Mol Sci 2019;20(11). PubMed PMID: 31174326. Pubmed Central PMCID: PMC6600154. Epub 2019/06/09. eng, DOl: https:/ doi.org/10.3390/ijms20112780.

48. Dibbell-Hope $S$. The use of dance/movement therapy in psychological adaptation to breast cancer. The Arts in Psychotherapy. 2000 2000/01/01/; 27(1):51-68.

49. Thieser S, Dörfler J, Rudolph I, Wozniak T, Schmidt T, Hübner J. Influence of ballroom dancing on fatigue, body image, self-efficacy, and endurance of cancer patients and their partners. Med Oncol. 2021;38(2): 15. 33507443, PMC7843482. Epub 2021/01/29. eng. https://doi.org/10.1 007/s12032-021-01459-0.

50. Oei SL, Thronicke A, Kröz M, von Trott P, Schad F, Matthes H. Impact of oncological therapy and Viscum album $L$ treatment on cancer-related fatigue and internal coherence in nonmetastasized breast cancer patients. Integr Cancer Ther. 2020;19:1534735420917211. 32478590, PMC7273755. Epub 2020/06/02. eng. https://doi.org/10.1177/1534735420917211.

51. World Medical Association Declaration of Helsinki: ethical principles for medical research involving human subjects. JAMA. 2013 Nov 27;310(20): 2191-4. PubMed PMID: 24141714. Epub 2013/10/22. eng.

\section{Publisher's Note}

Springer Nature remains neutral with regard to jurisdictional claims in published maps and institutional affiliations.

Ready to submit your research? Choose BMC and benefit from:

- fast, convenient online submission

- thorough peer review by experienced researchers in your field

- rapid publication on acceptance

- support for research data, including large and complex data types

- gold Open Access which fosters wider collaboration and increased citations

- maximum visibility for your research: over $100 \mathrm{M}$ website views per year

At $\mathrm{BMC}$, research is always in progress.

Learn more biomedcentral.com/submissions 